\title{
Epigrafía romana del Museo de Doña Mencía (Córdoba)
}

\section{Roman inscriptions of Museo de Doña Mencía (Córdoba)}

\author{
MaURICIO PAStOR MuÑOZ*
}

\begin{abstract}
RESUMEN
ABSTRACT

En este trabajo se analizan y estudian las inscripciones latinas de Doña Mencía (Córdoba), especialmente las que se conservan en el Museo HistóricoArqueológico de la localidad. De todas ellas, destacamos los epígrafes procedentes del yacimiento arqueológico denominado "El Laderón", una inscripción singular que podemos considerar como "la primera señal de tráfico de la Hispania romana", $y$, por último, un monumento epigráfico-funerario, inédito, que bien puede tratarse de una cupa.

PALABRAS CLAVE In this study are analysed and studied Latin inscriptions the Doña Mencía (Córdoba), especially those which are preserved in the Historical-Archaeological Museum at the town. Of all of them, we emphasize the epigraphs proceeding from the archaeological site called "The Laderón", a singular inscription that we can consider to be "the first traffic sign of Hispania", and, finally, an epigrafic-burial mound, an unprecedented monument, which it can be about a cupa.

$\begin{aligned} \text { PALABRAS CLAVE } & \text { KEYWORDS } \\ \text { Inscripciones latinas, Bética, Hispania, } & \text { Latin inscriptions, Baetica, Hispania, Doña } \\ \text { Doña Mencía. } & \text { Mencía. }\end{aligned}$
\end{abstract}

Recibido el 6 de junio de 2012. Aceptado el 5 de agosto de 2012

En memoria de nuestro inestimable «maestro epigráfico», G. Alföldy, ofrecemos este breve estudio de las inscripciones latinas conservadas en el museo histórico-arqueológico de la localidad cordobesa de Doña Mencía, donde tuvimos la ocasión de impartir una conferencia sobre el tema ${ }^{1}$.

* Catedrático de Historia Antigua de la Universidad de Granada. Dirección: Urb. "Jardines de Rolando", 48, 18011-Granada; e-mail: mpastor@ugr.es; móvil: 629452927.

1 Invitado por el Sr. Ángel Rodríguez Aguilera, director del Museo y Coordinador de las II Jornadas sobre Patrimonio; la conferencia fue impartida el 17/10/2009. El texto inédito, ha sido expresamente revisado y preparado para este trabajo. 
Las inscripciones latinas tienen un valor incalculable para la investigación de la sociedad y la historia romanas ${ }^{2}$. Como legado directo de la Antigüedad, como testigos «parlantes» de una cultura pasada que ha marcado la imagen de Europa de forma duradera, las inscripciones ofrecen una información fiable en las ruinas arqueológicas y señalan con frecuencia cual era la funcionalidad de los monumentos $^{3}$. Desde la Roma republicana, pero sobre todo a partir del reinado de Augusto y en lo sucesivo durante toda la época imperial hasta el s. VI d.C., las inscripciones latinas han acompañado la milenaria historia de Roma, de sus provincias y sus habitantes, reflejando como un medio omnipresente todas las facetas de la comunicación social.

Los procesos de investigación de los epigrafistas son muy diferentes: pueden recurrir a las excavaciones arqueológicas en los sitios privilegiados, pero también deben acudir a los epígrafes encontrados por azar o los descubiertos por excavadores clandestinos, o los que se conservan el los Museos provinciales o locales, como es el caso de las inscripciones que estudiamos aquí, puesto que, en ocasiones, existen inscripciones inéditas, desconocidas, o mal leídas, que necesitan una nueva lectura, interpretación o/y revisión ${ }^{4}$.

Como es bien sabido, las inscripciones latinas constituyen un inmenso arsenal de datos de las más diversas características. En concreto, permiten conocer detalles sobre la sociedad, administración municipal, religión, instituciones, ejército, etc., aparte de ofrecernos los nombres y apellidos (nomina et cognomina) que llevaron los habitantes de localidades como Doña Mencía durante la época romana. A través de las inscripciones se pueden deducir, a grandes rasgos, cuales eran sus familias más representativas y quiénes de sus miembros destacaron, no sólo en su propio municipium, sino también en su proyección social exterior ${ }^{5}$

El yacimiento arqueológico más conocido del término municipal de Doña Mencía se denomina «El Laderón», ubicado sobre un promontorio alargado, en

2 Nadie mejor que el aquí homenajeado puede ilustrarnos sobre la importancia de lo que decimos. Cf. ALFÖLDY, G., "La cultura epigráfica de la Hispania romana: inscripciones, auto-representación y orden social" en Hispania. El Legado de Roma, Zaragoza, 1998, pp. 289-301.

3 Cf. al respecto, ENCARNAÇAO, José d', Epigrafía. As Pedras que Falam, Coimbra (Portugal), 2006. Vid. también, MAC MULLEN, R., "The Epigraphic Habit in the Roman Empire", The American Journal of Philology, 103, 3, 1982, pp. 233 ss.

4 En todas las épocas, desde el Renacimiento a nuestros días, se han venido haciendo comentarios y revisiones de los textos epigráficos. Sólo voy a citar la nueva revisión del Corpus Inscriptiones Latinarum (CIL), de la que tenemos el honor de participar en el CIL II², del que hasta ahora se han publicado tres volúmenes (V, VII y XIV), que corresponden al Conventus Astigitanus, Cordubensis y Tarraconenses respectivamente; precisamente, algunas de las inscripciones relacionadas con Doña Mencía se recogen en el Astigitanus (CIL, II2/5.342-347).

5 Todos estos aspectos y otros similares podemos verlos en cualquier manual de Epigrafía Latina. En los últimos años han aparecido varios, de los que solamente voy a mencionar los siguientes: P. CORBIER, L'Epigraphie Latine, París, 1998 (traducción en castellano y apéndice: PASTOR, M., "Epigrafía Latina, Granada, 2004); SCHMIDT, M. G., Einführung in die Lateinische Epigraphik, Darmstadt, 2004; LASSÉRE, J. M., Manuel d'Épigraphie Romaine. L'individu-la cité, París, 2005; ANDREU, J. (Coord.), Fundamentos de Epigrafía Latina, Madrid, 2009 (con bibliografía complementaria). 
una de las estribaciones del Cerro de Camarena, de la Sierra de Cabra ${ }^{6}$. Es casi inaccesible por el sur y por el Arroyo de la Plata al Oeste, y domina el paso de un antiguo camino prehistórico, que seguramente fue el origen de la vía romana de Jaén (Aurgi) a Aguilar de la Frontera, (Ipagrum), ahora llamada «Camino de Metedores», que se dirigía desde Obulco por Ipsca, Iponoba, Igabrum y por Monturque hasta el vado del Genil situado en Puente Genil (de allí procede una inscripción romana que luego veremos y un fragmento de un miliario, hoy perdido, citado por Hubner). La vía seguiría el camino natural de Baena a Cabra por el valle del Marbella y los cimientos de las caídas casi verticales de la Sierra de Camarena, aunque no esta claro donde enlazaría con la vía principal, si en Ipagrum (Aguilar de la Frontera) o en Monturque, verdadera encrucijada de caminos y donde han aparecido abundantes sepulturas sin ajuares, ni inscripciones, pero que indican su situación junto al camino, con la intención de mantener el acceso a las tumbas a través de las vías publicas ${ }^{7}$.

El cerro de «El Laderón» está lleno de fortificaciones a un lado y a otro, como el Cerro del Mingillar, el cerro Cotillas, Oreja de la Mula, Castillejo de Luque, Castillo de Sueros, Fuente del Carmen, el Majuelo, y al otro lado del Arroyo, el cerro la Plata, la Majá del Serrano, que parecen ser un bastión defensivo de la ciudad, como podemos apreciar, por ejemplo, en esta fotografía (Fig. 1).

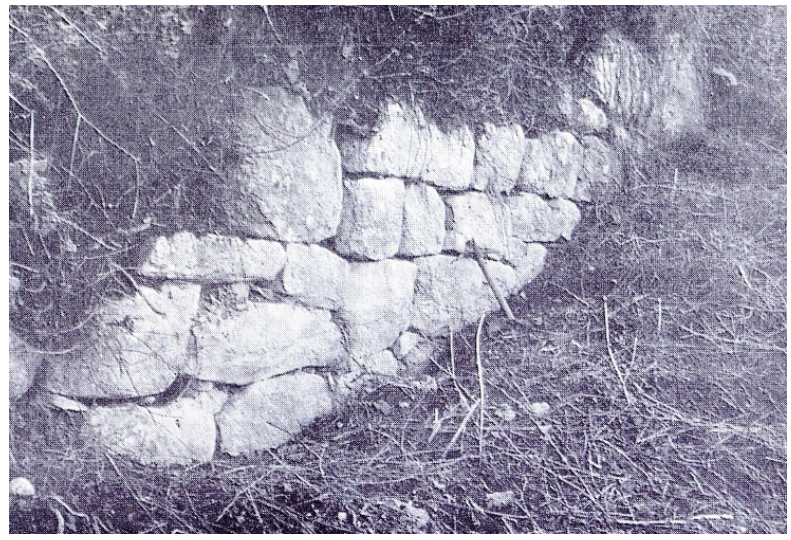

Fig. 1. Restos de fortificaciones de "El Laderón" (Foto de J. Fortea- J. Bernier)

6 Sobre el yacimiento, cf. principalmente, FORTEA, J. y BERNIER, J., Recintos y fortificaciones ibéricos en la Bética, Salamanç, 1970, pp. 49-51, Lám. XIII, 1; BERNIER, J., SÁNCHEZ ROMERO, C., JIMÉNEZ URBANO, J. y SÁNCHEZ ROMERO, A., Nuevos yacimientos arqueológicos en Córdoba y Jaén, Córdoba, 1981, pp. 96-101.

7 Cf. SEGURA ARISTA, L., La ciudad íbero-romana de Igabrum, Cabra, Córdoba, Córdoba, 1988, pp. 130-134. 
J. Fortea y J. Bernier ya indicaron en su momento que, «el cerro es de pequeña extensión, y debió tener una vida intensiva desde los tiempos prehistóricos hasta la época visigoda y árabe como se deduce de los objetos recuperados en las prospecciones realizadas: hachas de piedra pulimentada, cerámicas griegas, campanienses, ibéricas, romanas, así como monedas ibéricas y romanas, y fragmentos de esculturas ibero romanas, que se han recuperado continuamente de las laderas del cerro, donde proliferan los restos arqueológicos de diversas épocas históricas ${ }^{8}$.

Hasta las investigaciones que realizaron en el oppidum J. Bernier, J. Fortea y los jóvenes del Grupo de Montaña de Doña Mencía, dirigido por D. C. Sánchez Romero, que tanto ha hecho por la investigación histórico-arqueológica de la comarca, se creía que tan solo tenia las murallas romanas que estaban a la vista, pero sus actuaciones pusieron al descubierto el recinto entero, con grandes sillares que se adaptan a la forma del montículo que esta perfectamente construido. Lamentablemente, no se han llevado a cabo excavaciones arqueológicas sistemáticas, tan solo pequeñas catas en busca de vestigios y objetos, por lo que el yacimiento está bastante deteriorado. Se necesitarían nuevas excavaciones para poner en valor todo el potencial que el cerro encierra. No obstante, tanto el cerro de «El Laderón», como la cercana «Plaza de Armas» de Nueva Carteya, permiten datar perfectamente las murallas, que no irían mas allá de los comienzos de la romanización, tal vez, desde los inicios del siglo II a.C. Llama la atención la gran riqueza de las cerámicas recuperadas en el cerro, que van desde el siglo $\mathrm{V}$ a. $\mathrm{C}$. hasta la época alto imperial (siglo I d. C.).

A pocos kilómetros al Noroeste se encontraría la ciudad ibérica que había en «El Laderón», sobre un antiquísimo camino, que se dirige a Málaga por un ramal y por otro a Ipagrum. Ésta seria la Vía Gades-Castulo, tal vez la vía a la que se refiere la inscripción número 2 , que veremos más adelante. Todos estos recintos fortificados servirían para defender las comunicaciones que iban por el valle.

Lo dicho con anterioridad permite pensar que el recinto fortificado de «El Laderón» constituyó una ciudad ibérica de cierta importancia, que seria romanizada mas tarde cuando se impusieron en todo el territorio las tropas romanas, como demuestran no solo las cerámicas recuperadas, sino también los restos de inscripciones procedentes de este yacimiento y de sus inmediaciones.

Sin embargo, desconocemos el nombre ibérico o romano que debió tener la ciudad, a pesar de que algunos investigadores han querido identificarla con la Vesci Faventia, citada por Plinio ${ }^{9}$, como una de las ciudades estipendiarias del Conventus Astigitanus. Vesci la cita también Ptolomeo, ubicándola entre Ilipula Laus y

8 FORTEA, J. y BERNIER, J., Op. Cit. p. 50.

9 PLIN. Nat. Hisp. III, 10. Cf. FERNÁNDEZ GUERRA, A., Arqueología cristiana, Madrid, 1876, p. 399; SCHULTEN, A., Fontes Hispaniae Antiquae, III, (Las guerras de 237-154 a. d. J.C.), Barcelona, 1935, p. 197; THOUVENOT, R., Essai sur la province romaine de la Betique, París, 1940, p. 110; TOVAR, A., Iberische Landeskunde, I, Baetica, Baden Baden, 1974, p. 62; BERNIER, J. et alii, Op. Cit. p. 98. 
Osqua ${ }^{10}$. Igualmente se refiere a ella Tito Livio, que la menciona junto a Helos, como los dos oppida conquistados por el pretor M. Fulvio en la Bética ${ }^{11}$; y también aparece mencionada en varias monedas con la leyenda $\mathrm{VESCI}{ }^{12}$. Actualmente, los historiadores no aceptamos su identificación en el cerro de «El Laderón», donde se ha ubicado sin fundamento alguno, aunque no se pueda descartar ninguna hipótesis. Por ejemplo, M. Pastor situó Vesci Faventia en la «tierra de Loja», bien en Huétor Tajar, bien en el «Cerro de la Mora» de Moraleda de Zafayona, donde las excavaciones realizadas evidenciaron la existencia de un poblado ibero-romano de enorme importancia ${ }^{13}$.

Respecto a las inscripciones del Museo de Doña Mencía, tenemos las siguientes:

1. Fragmento de inscripción honorífica. Parte de un pedestal cilíndrico, de caliza roja, de la conocida con el nombre de «mármol de Cabra» ${ }^{14}$, roto por todas partes. Fue descubierto en una fecha anterior a 1945 en una colina al norte del oppidum de «El Laderón»; 27 x 23. Letras: $6 \mathrm{~cm}$, primera línea y 3 las de la segunda. Se aprecia una ínterpunción triangular.

Actualmente se conserva en el Museo Arqueológico Provincial de Córdoba con el número de inventario 24249 c. Allí la vimos y fotografiamos en 2009, aunque la fotografía que ofrecemos es la del CIL, II²/5,341 (Fig. 2).

10 PTOLOM. II, 4, 9; Osqua se ha localizado en el "Cerro de León”, en Villanueva de la Concepción, cerca de Antequera; vid. CIL, II, 2007; CIL, II², XXXI, pp. 201-203; TOVAR, A., Op. Cit. p. 134.

11 LIV. Ab urbe condita, XXXV, 22, 5: et in utraque Hispania eo anno res prospere gestae, nam et $C$. Flaminius oppidum Licabrum munitum opulentumque vineis expugnavit et nobilem regem Corribilionem vivum cepit et $M$. Fulvius procónsul cum duobus exercitibus hostium duo secunda proelia fecit oppida duo Hispanorum Vesceliam Helonemque et castella multa expugnavit, alia voluntate ad eum defecerunt, Vid. ut supra, n. 9.

12 HÜBNER, E. (ed.), Monumenta Linguae Ibericae (MLI), Berlín, 1893, p. 123; VILLARONGA, L., Numismática antigua de Hispania, Barcelona, 1979, p. 179; GUADÁN, J. M., Numismática ibérica e ibero-romana, Madrid, 1969, p. 183.

${ }^{13}$ Cf. PASTOR, M., CARRASCO, J. y PACHÓN, J. A., "Cerro de la Mora (Moraleda de Zafayona, Granada)", Noticiario Arqueológico Hispánico, XII, 1981, pp. 137-168; CARRASCO, J., PASTOR, M. y PACHÓN, J. A., "Cerro de la Mora (Moraleda de Zafayona, Granada). Memoria de la campaña de excavaciones de 1979", NAH, XIII, Madrid, 1983, pp. 5-161; CARRASCO, J., PASTOR, M. y PACHÓN, J. A. "Cerro de la Mora. Moraleda de Zafayona. Resultados preliminares de la segunda campaña de excavaciones (1981). El Corte 4", Cuadernos de Prehistoria de la Universidad de Granada, 6, 1981 (1984), pp. 307-354; PASTOR, M., Indigenismo y romanización en la tierra de Loja, Granada, 1986, p. 223; PASTOR, M., "Las tierras granadinas hace 2.000 años", Revista de la C.E.C.E.L., 2, Valencia, 2002, p. 309.

14 Sobre el tipo de mármol, cf. principalmente, CANTO, A., "Avances sobre la explotación del mármol en la España romana”, Archivo Español de Arqueología, 50-51, 1977-1978, pp. 175 ss.; SEGURA ARISTA, L., Op. Cit. pp. 112-130; concretamente sobre las lápidas de Cabra y sus alrededores, vid. STYLOW, A., "Inscripciones latinas del Sur de la Provincia de Córdoba", Gerión, I, 1983, pp. 267-303; PUERTA, C. y STYLOW, A., "'Inscripciones romanas del sureste de la Provincia de Córdoba", Gerión, 3 , 1985, pp. 117-346. 


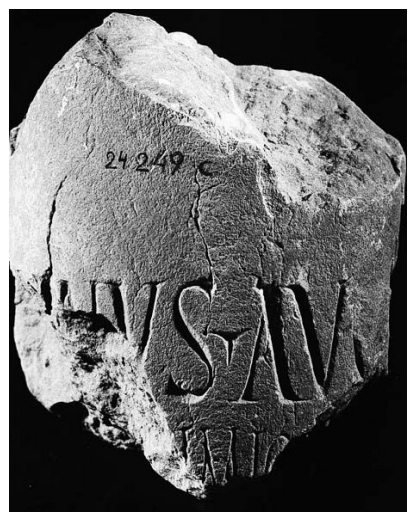

Fig. 2. Inscripción de «El Laderón» (Fotografía de CIL, I²/5, 341)

L. Segura pensó que se trataba de un miliario ${ }^{15}$, pero no lo parece en absoluto, de aquí que propongamos la siguiente lectura:

[-- - - - ]

- - - IMIVS · AVG

- - IMIO F

[-- - - - ]

- Trascripción: [P(ublius) Sept]imius · Aug(usti) [lib(ertus) -?

- - - cum P(ublio) Sept]imio f(ilio) - - -

- - d(edit) $d$ (edicavit)]

- Traducción: Publio Septimio, liberto de Augusto, junto con su hijo Publio Septimio lo donó y dedicó...?

- Bibliografía: A. Sánchez Romero en J. Bernier et alii, Op. Cit. p. 99 con fotografía; L. Segura Arista, Op. Cit. p. 132, n. 237, que cree que es un miliario; CIL, ${ } \mathrm{I}^{2} / 5,341$, donde A. Stylow, a modo de ejemplo, interpreta el texto como [-Septi]mius Auc[tus] - - - cum Sept]imio.

- Comentario: Probablemente se trata de una inscripción honorífica, aunque no lo sabemos con seguridad, por lo que hay que incluirla entre las de incerti generis. La primera y la última línea están perdidas. La lectura se ha realizado atendiendo a que es posible ver restos de las letras IMIVS - AVG y de IMIO F. En la fractura de la derecha parece verse una F longa hasta rozar el lado izquierdo de la G superior.

Las letras son capitales cuadradas de buena factura, por lo que parece que se trata de un liberto imperial, el cual, junto con su hijo, del mismo nomen, $P$ (ublius) Septimius, dedicaron este monumento por algo relacionado con el culto imperial.

15 SEgurA ARISTA, L., Op. Cit. p. 132, n. 237. 
La forma redondeada superior impide asegurar que la pieza se usara como pedestal, aunque podría estar situada en el recinto exterior de algún templo o edificio público, o tal vez, en el foro del municipio. No obstante, por esta inscripción, podemos conjeturar que en el municipio ibero-romano de «El Laderón» se rendía culto al emperador, al igual que en otros municipios de la Bética.

Por la forma y caracteres de las letras puede datarse a finales del siglo II ó comienzos del III de nuestra era.

2. Inscripción indicativa. Cipo de piedra caliza de color blanco, de la denominada «crema de Cabra» ${ }^{16}$. El campo epigráfico, sin definir, se encuentra en la parte frontal, que está pulimentada, mientras que la parte posterior y los laterales están en basto. Su estado de conservación es bastante bueno, aunque está roto por abajo y por las esquinas inferiores; (35) x 24/21 x 7/8,5. Letras entre 3 y 3,5 cm y son capitales librarias (librariae) ascendentes, de buena factura. Lleva interpunciones de forma triangular y al final termina con una hedera.

Fue encontrado en 1964 en la finca «Llano Medina», situada a un kilómetro y medio aproximadamente de Doña Mencía, en el yacimiento llamado «Henazar», entre unas sepulturas excavadas junto a la calzada romana que pasaba por ese lugar y que, como hemos dicho, enlazaba Iponoba (Baena) con Igabrum (Cabra), cerca también de «El Laderón», donde se encontraba una ciudad ibero-romana de nombre desconocido y cercana también al asentamiento de "Monte Horquera», la Nueva Carteya, fundación del siglo XIX, que adquirió este nombre en base a la gran abundancia de restos arqueológicos, epígrafes y obras de arte que allí se encontraron ${ }^{17}$.

Actualmente se conserva en el Museo Arqueológico de Córdoba con el nº de inventario: 24249. Una copia del original, en escayola, se encuentra en el Museo de Doña Mencía. Ambas las vimos y fotografiamos en 2009, aunque la foto que acompaña es de A. Rodríguez Aguilera (Fig. 3):

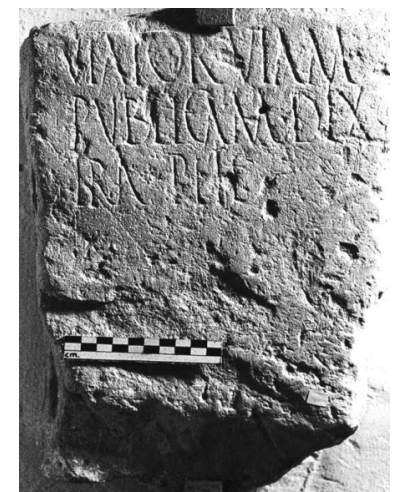

Fig. 3. Inscripción de «posible señal de tráfico» (Fotografía de A. Rodríguez Aguilera)

16 Ut supra. Vid. la nota 14.

17 Cf. CIL, II²/5,348-365; Cap. XIII. "Monte Horquera”, pp. 96-98. 
En el texto se puede leer lo siguiente:

VIATOR · VIAM

PVBLICAM · DEX

TRA P PETE (hedera)

- Trascripción: Viator, viam / publicam, dex/tra, pete

- Traducción: «Caminante, dirígete a la vía pública, por la derecha».

- Bibliografía: F. J. Fernández Nieto, J. Fortea y J. M. Roldán, «Una nueva inscripción del Museo Arqueológico de Córdoba», Zephyrus, XIX-XX, 1968-1969, pp. 169-173 (AE, 1969/1970, n. 254; E. Melchor, «Sistemas de financiación y medios de construcción de la red viaria hispana», Habis 23, 1992, p. 136); J. Bernier et alii, Op. Cit. p. 60, n. 67 y p. 61, n. 70 (R. Corzo y M. Toscano, Vías romanas en Andalucía, Sevilla, 1992, p. 225, n. 6); D. Ortiz Juárez et alii, Catálogo artístico monumental de la Provincia de Córdoba, Córdoba, 1981, III (1985), pp. 98-99; P. Silliéres, Les voies de communication de l'Hispanie méridionale, París, 1990, p. 166, n. 110; CIL, II²/5,343.

- Comentario: Esta curiosa inscripción, de carácter privado, nos informa del camino que debía seguir el viajero que había llegado hasta allí. Por su contenido puede ser considerada como «la primera señal de tráfico de la epigrafía latina de Hispania», e incluso, de la historia romana, puesto que, hasta este momento, no había aparecido otra con texto similar. Es evidente que se trata de un cipo admonitorio para el viajero, al que se le indica que se dirija a la «vía pública» por la derecha. Probablemente, la vía pública a la que se refiere la inscripción sea el camino que hemos mencionado antes, que enlazaba Iponoba con Igabrum. El cipo debía estar ubicado a la salida de alguna localidad o de algún cementerio y en alguna encrucijada de caminos, porque sino la advertencia no habría sido necesaria.

El termino pete, del verbo peto, «ir», «dirigirse a», «caminar hacia», parece claro, sin necesidad de buscar cualquier otra interpretación mas complicada, como hicieron F. J. Fernández Nieto et alii, que leyeron, erróneamente, dextro pede ${ }^{18}$ (con el pie derecho).

Por la forma de las letras y los caracteres paleográficos se puede fechar a finales del siglo I ó comienzos del II, aunque hay autores que la datan a finales del II y comienzos del III.

3. Inscripción funeraria. Fragmento de placa de mármol blanco con borde irregular original por arriba y por la izquierda y por abajo, borde liso. Está roto por la derecha, aproximadamente por la mitad. La parte posterior esta alisada, al igual que la frontal, donde se encuentra el texto; $19,5 \times 14 \times 2,5$. Letras de 3,5 cm en la primera línea y 2,2 en las siguientes. En la parte inferior se aprecian líneas-guía y las interpunciones son de sección triangular; tras la $\mathrm{D}$, de la primera línea, aparece una hedera muy estilizada.

18 FERNÁNDEZ NIETO, F. J. et alii, Op. Cit. pp. 171-173. 
Apareció en 1965 en el «Cortijo Nuevo», en el término municipal de Espejo, asociada a cerámicas sigillatas y a dos monedas imperiales en mal estado de conservación e ilegibles. Muy cerca, en la zona del Puerto, a un kilómetro y medio de Doña Mencía, situado en el camino de Metedores, también apareció un candelabro de vidrio ${ }^{19}$.

Actualmente se conserva en el Museo Histórico Arqueológico de Doña Mencía, con el no de inventario 84/1, donde la vimos y fotografiamos en 2009 (Fig. 4):

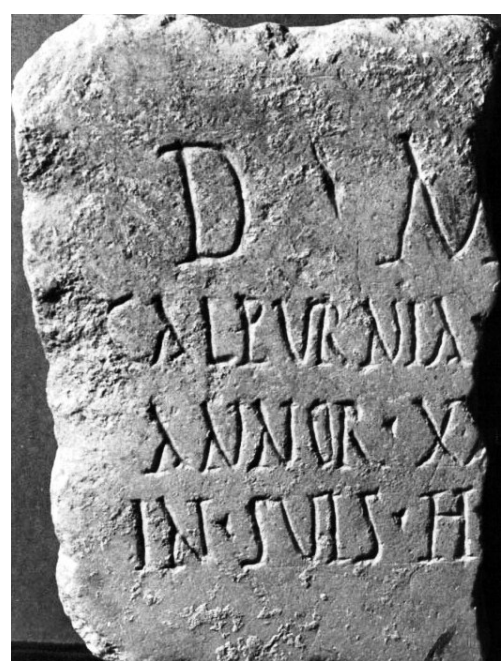

Fig. 4. Inscripción de Calpurnia. Museo de Doña Mencía (Foto de M. Pastor)

En el texto se lee lo siguiente:

$\mathrm{D} \cdot($ hedera $) \mathrm{M}[\cdot \mathrm{S} \cdot]$

CALPVRNIA $\cdot[--\cdot]$

ANNOR $\cdot$ XX [- - PIA]

IN $\cdot$ SVIS $\cdot \mathrm{H} \cdot[\mathrm{S} \cdot \mathrm{E} \cdot \mathrm{S} \cdot \mathrm{T} \cdot \mathrm{T} \cdot \mathrm{L} \cdot]$

- Transcripción: D(is) M(anibus) [S(acrum)] / Calpurnia [- - - ] / annor(um) XX [pia] / in suis, h(ic) [s(ita) e(st) s(it) t(ibi) t(erra) I(evis)].

- Traducción: «Consagrado a los dioses Manes. Calpurnia ...? de 20 ? años de edad, aquí esta enterrada. Sea para ti la tierra ligera».

${ }^{19}$ BERNIER, J., et alii, Op. Cit. pp. 99-100. 
- Bibliografía: A. Stylow, «Inscripciones latinas del Sur de la Provincia de Córdoba», Op. Cit. pp. 287-289, n. 5 (AE, 1983, 537); D. Ortiz Juárez et alii, III (1985), p. 99; CIL, II/2/5,345.

- Comentario: Las letras son capitales actuarias o rústicas (librariae) y muestran unos rasgos muy marcados: curvatura en los trazos verticales de la $\mathrm{N}$ y la $\mathrm{V}$, casi idénticas; la $\mathrm{O}$ es casi rectangular y las $\mathrm{X}$ llevan el punto de cruce muy bajo; la $\mathrm{H}$ lleva el trazo trasversal muy alto y las A muy abiertas con el trazo oblicuo.

El texto de la inscripción debió estar centrado alrededor de un eje central que pasaba por la $\mathrm{M}$ de la formula onomástica del inicio (D. M. S.). Esto significa que el cognomen de la difunta, que no se ha conservado era mas o menos igual de largo que el gentilicio, a lo que habría que añadir su filiación, o patronazgo, en caso de que fuera una liberta, que también seria factible. El gentilicio, Calpurnius/Calpurnia es uno de los más frecuentes en Hispania y concretamente en la Betica ${ }^{20}$.

Se trata, por tanto, del epitafio de una mujer, cuya edad no sabemos con certeza, pues el numeral completo no se ha conservado, de la gens Calpurnia. Su cognomen tampoco se ha conservado. No obstante, se trata de un testimonio evidente del culto a los dioses Manes en estas tierras, donde se han encontrado varios testimonios con esta misma formula funeraria de devoción a los dioses de los difuntos ${ }^{21}$. En consecuencia, es uno mas de los nombres personales (nomina) de las gentes que habitaron estas tierras cordobesas.

Por la forma y caracteres de las letras y por la aparición de las formulas típicas de las inscripciones funerarias (DMS, HSE, STTL), hay que datar este epígrafe a partir de la segunda mitad del siglo II de nuestra era.

4. Inscripción funeraria. Apareció en un lugar cercano a Doña Mencía, como informan algunos manuscritos antiguos. Según ellos, era una inscripción funeraria sobre una piedra de mármol blanco, de "dos tercias de alto y una de grueso»"22, es decir, $56 \times 28 \times(i)$.

Se encontró en 1668 «encima de un sepulcro muy grande, en el pago de la Dehesa del Puntal, 3 cuartos de legua de la villa (Cabra), camino de Baena, dentro de la casería de D. Pedro de Heredia y Cabrera Ponce de León „23. Dicho predio se encontraba aproximadamente a 14 kilómetros de Cabra y a 5 de Doña Mencía. En la actualidad está perdida o en paradero desconocido.

El texto decía lo siguiente:

TERTVL[L]A

\section{ANNORVM}

20 ABASCAL, J. M., Los nombres personales en las inscripciones Latinas de Hispania, Murcia, 1994, pp. 104-106., con más de 150 representantes de esta familia.

21 Sobre el culto a los Manes en la Bética, cf. principalmente, PASTOR, M., "Los dioses Manes en la epigrafía funeraria bética", Mainake, XXVI, Málaga, 2004, pp. 381-394.

22 VEGA MURILLO Y AGUILAR, J. DE, Op. Cit. 19 r; CIL, II, 1623; CIL II2/5, 346.

23 Ibidem 
$\mathrm{XVI} \cdot \mathrm{HIC}$ SITA

\section{EST}

- Trascripción: Tertul(I)a / annorum / XVI. Hic sita / est

- Traducción: «Tertulla, de 16 años de edad, aquí esta enterrada».

- Bibliografía: J. de Vega Murillo y Aguilar, Historia...Egabra, ms. Biblioteca Nacional de Madrid, Sign. G 183, ms. 1668, 19 r; (E. Hubner, CIL, II, 1623 = ILER, 2552 = L. Segura, Op. Cit. p. $179=\mathrm{CIL}$, II2/5,346); N. Albornoz y Portocarrero, Historia de la ciudad de Cabra, Madrid, 1909, 34.

- Comentario: Nexo en VM de annorum. Tradicionalmente se ha leído el nombre de la difunta como Tertulia, en vez de Tertulla. El nomen lo corrigió Hubner acertadamente, puesto que Tertulla, con dos eles, es un cognomen frecuente entre los esclavos y libertos de la Betica ${ }^{24}$. Este mismo nombre se ha documentado en una inscripción granadina ${ }^{25}$. El nombre, según Kajanto, esta basado en el orden del nacimiento y es característico en el ámbito de esclavos y libertos ${ }^{26}$. Probablemente, en este caso lo llevaba una joven de 16 años, Tertulla, una sierva. Este dato, de ser cierto, nos documentaria en este lugar la existencia de esclavos que servirían en alguna de las familias destacadas de los municipios cercanos. El mismo hecho de llevar un solo nombre nos está indicando su condición servil.

Por la forma de las letras y por el tipo de mármol, la inscripción se ha datado a finales del siglo II ó comienzos del III.

5. Inscripción funeraria. Estela arenisca, redondeada por la parte superior. No está alisada, sino que se presenta en basto por todas partes. Su estado de conservación es muy malo. Del texto solo conservamos la primera línea y ligeros rasgos de una segunda línea, aunque tuvo que tener más texto, pero, hoy, ya no se conserva; 115 57/64 × 38. Está bastante desgastada ya que cuando se encontró medía mucho más (165 x 65 x 60). Letras entre 7,5/9 cm Los puntos son de sección triangular.

Se encontró aproximadamente en 1903 «en la margen derecha del río Guadalmoral, terreno de Rivillas, delante de una casa de campo perteneciente a las hijas de D. Alfonso Villareal junto con grandes piedras berroqueñas labradas» 27 . Durante bastante tiempo se pensó que se había perdido, pues no se tenia noticia de ella, hasta que apareció de nuevo en 1985, junto con muchas piedras cuadradas, que formaban parte de las paredes de las sepulturas, en la Casería Nueva (Baena), a unos tres kilómetros de Doña Mencía, en la orilla izquierda del río Guadalmoral, y desde allí fue trasladada al Museo de la localidad, donde la vio y fotografió A. Stylow en $1985^{28}$.

24 ABASCAL, J. M., Op. Cit. p. 526.

25 PASTOR, M., Corpus de Inscripciones Latinas de Andalucía. Volumen IV: Granada, Sevilla, 2002, n. 22.

26 KAJANTO, I., The Latin Cognomina, Helsinki, 1965, p. 292.

27 VALVERDE PERALES, F., Op. Cit. pp. 48-49.

$28 \mathrm{CIL}, \mathrm{II}^{2} / 5,347$, p. 94. 
Actualmente se conserva en el Museo Histórico y Arqueológico de Doña Mencía, donde la vimos y fotografiamos en 2009 (Fig. 5):

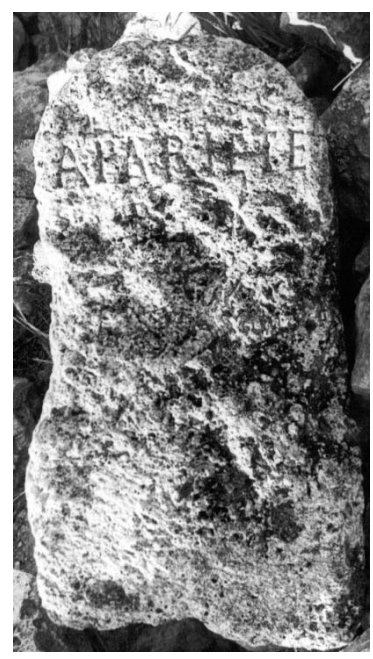

Fig. 5. Inscripción funeraria (Fotografía M. Pastor)

Solamente se leen las siguientes palabras:
A · PARIETE
$P \cdot X$

- Trascripción: A pariete / p(edes) X/ - - -

- Bibliografía: F. Valverde Perales, Historia de la villa de Baena, Toledo, 1903, pp. 48-49, que leía: APARIEII / P X / C / E / F, pero hoy día no se aprecia absolutamente nada de las tres ultimas líneas, ni siquiera las incisiones; CIL, II2/5,347.

- Comentario: Las letras son librariae bien formadas. Al parecer, aparecieron dos estelas gemelas y ambas formando parte de los muros de las sepulturas, una en el lado izquierdo y otra en el derecho del río situado entre Nueva Carteya y Doña Mencía, que fluye por la región de las Rivillas. Pero de las dos, únicamente se ha conservado ésta.

En consecuencia, estamos ante una inscripción indicativa que señalaba el lugar donde estaba situada la sepultura y la distancia que había desde el muro hasta ella; en el caso que nos ocupa, concretamente se encontraba a diez pies. Lamentablemente, no podemos saber las otras distancias por no haberse conservado el resto del texto, o por haber desaparecido la otra estela en la que estaría escrita la otra distancia.

Por el tipo y características de las letras se ha fechado en torno al siglo I. 
6. Inscripción funeraria. Fragmento de losa o bloque de piedra caliza. La pieza original debió ser cuadrada, pero solo conservamos una esquina de la parte inferior. Está rota por arriba, por abajo y por la derecha. La parte frontal está pulimentada, por detrás, en basto. Lo conservado mide (28) x 34 × 12/14. En la parte inferior izquierda presenta un agujero circular producto de su reutilización posterior como umbral de una puerta. Las letras miden 5 ' $5 \mathrm{~cm}$. La única interpuncion que se aprecia es de sección triangular.

Apareció en 1967 en el yacimiento denominado «Cortijo Nuevo» en el término municipal de Espejo (Córdoba), la antigua Ucubi29.

Actualmente se conserva en el Museo Histórico Arqueológico de Doña Mencía con el no de inventario 30, donde la vimos y fotografiamos en 2009 (Fig. 6):

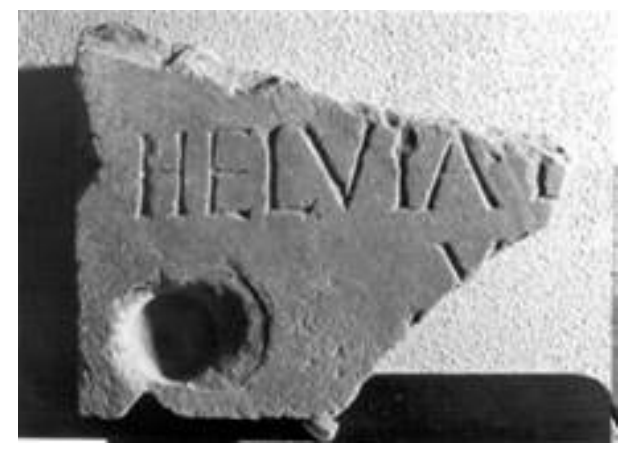

Fig. 6. Inscripción de Helvia. Museo de Doña Mencía (Fotografía M. Pastor)

Podemos leer lo siguiente:

[- - - - ? ?

HELVIA · L [-- - ]

$\mathrm{X}[---]$

- Trascripción: - - - -? / Helvia L [- - -] / X [- - - ]

- Bibliografía: A. U. Stylow, «Beiträge zur lateinischen Epigraphik im Norden der Provinz Córdoba, II, Baedro, III, Mellaria«, MDAI(M), 28, 1987, p. 116 (AE, 1987, 553; HEp., 2, 1990, 327); CIL, II2/5,453.

- Comentario: Las letras son capitales cuadradas de mala factura. Detrás del nomen Helvia puede leerse una $L$ o una $E$, pero por la época de la inscripción, que hay que datar en el siglo I, concretamente en época de Augusto, es preferible una

${ }^{29}$ Cf. Colonia Claritas Iulia. Cf. CIL, II²/5,440-473; Cap. XVIII. “Vcub», pp. 118-126. 
$\mathrm{L}$ seguida de $\mathrm{F}$ de f(ilia), o incluso de $\mathrm{L}$ de I(iberta) y tras ella, el cognomen. Los rasgos de la $X$ si me parecen bastante claros. Podrían corresponder a un numeral o a la $X$ de $[V] X[O R]$.

Lamentablemente es muy poco lo que podemos comentar de esta inscripción, puesto que solo se puede leer el nomen de una femina, probablemente perteneciente a la gens Helvia. El nomen Helvia-Helvius es relativamente frecuente en las inscripciones hispanas, especialmente en Lusitania ${ }^{30}$.

7. Inscripción funeraria. Fragmento de una losa de piedra caliza oolitica, de color amarillento y muy blanda, probablemente de la modalidad denominada "crema de Cabra» ${ }^{31}$, de la que se conserva solamente el borde superior. Está rota por todas partes. La parte frontal, alisada, lleva el texto, la posterior está en basto. Lo conservado mide (35) × (34) x 4/8. Las letras, grabadas con una incisión profunda, miden $7 \mathrm{~cm}$ con un espacio interlineal de $9 \mathrm{~cm}$. La interpuncion es de sección triangular.

Se descubrió casualmente en 1965 en el yacimiento arqueológico de «Pasada Valera» del término municipal de Cabra, a unos 5 kilómetros al suroeste de Doña Mencía. Junto a ella aparecieron también algunos fragmentos de cerámica de terra sigillata, así como algunas monedas del Alto Imperio ${ }^{32}$.

Actualmente se conserva en el Museo Histórico-Arqueológico de Doña Mencía, con el $\mathrm{n}^{\circ}$ de inventario 62/1, donde la vimos y fotografiamos en 2009 (Fig. 7):

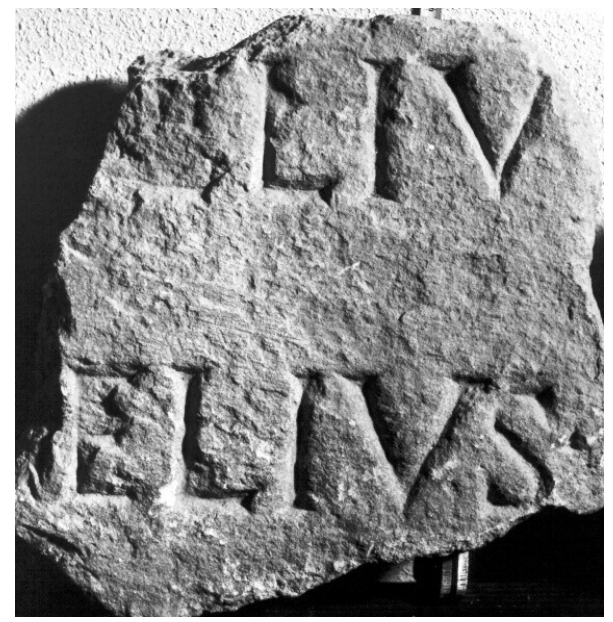

Fig. 7. Inscripción de [Corn]elius. Museo de Doña Mencía (Fotografía de M. Pastor)

\footnotetext{
${ }^{30}$ ABASCAL, J. M., Op. Cit. pp. 148-149.

${ }^{31}$ Ut supra. Vid. la nota 14.

32 BERNIER, J. et alii, Op. Cit. pp. 96 ss.
} 
Tan solo podemos leer lo siguiente:

[- - - - ]

[- - ] ELIVS · [- - - ]

[- - - COR]NELIVS · [- - -

[- - - - - ]

- Trascripción: [- - -]/[Corn]elius · [- - -]/[-Cor]nelius · [- - -]

- Bibliografía: A. Stylow, «Inscripciones latinas del Sur de la Provincia de Córdoba», Op. Cit. pp. 291-292, n. 7 (AE, 1983, 539; L. Segura, Op. Cit. p. 180, n. XXIII); CIL, II2/5,336.

- Comentario: Las letras son capitales cuadradas del siglo I d. C., probablemente de la primera mitad, fecha en la que hay que datar esta inscripción. En la línea 1 solo quedan restos de la curvatura superior de la $S$ final en la misma rotura de la piedra. El nomen es bastante claro, [Corn]elius, aunque tampoco podemos excluir otros muchos nombres latinos que terminan en -elius, como [A]elius, [Aur]elius, [Av]elius, etc. Sin embargo, en la línea 2, delante de la $\mathrm{E}$, en una zona muy desgastada, se aprecian pequeños restos de un trazo vertical y de otro oblicuo, que casi se tocan, por lo que parece probable que sea una N. En el municipio de Igabrum (Cabra) están atestiguados ambos nomina, el de la gens Aelia (M(arcus) Aelius M(arci) fil(ius) Níger, Aed(ilis), de época de Vespasiano ${ }^{33}$; y el de la Gens Cornelia (M(arcus) Cornelius A(uli) f(ilius) Nova[tus], Baebius, Balbus, praefectus fabrum, flamen provin(iae) Baeticae, de época de Trajano o un poco posterior $^{34}$, por lo que cualquiera sería posible ${ }^{35}$.

A. Stylow piensa que este fragmento formaría parte de una inscripción funeraria, en la que se nombrarían varios individuos con el mismo gentilicio, uno debajo del otro, por lo que tendríamos una inscripción funeraria temprana con una sucesión de los nombres, sin formulario funerario, que cubriría una tumba de los Cornelii que habían habitado una villa rustica situada entre Cabra y Doña Mencía ${ }^{36}$.

8. Inscripción indeterminada. Fuste de columna de piedra arenisca, del que tan solo conservamos la mitad aproximadamente; 54 x 34 . Letras entre 5 y $6 \mathrm{~cm}$.

Fue encontrado en 1969 en el yacimiento arqueológico denominado «Fuente del Río» del término municipal de Doña Mencia, donde apareció asociado a los niveles romanos con otras basas de columnas que se obtuvieron de las labores de limpieza que se hicieron en el castillo ${ }^{37}$. Su estado de conservación es

$33 \mathrm{CIL}, \mathrm{II}^{2} / 5,308$.

${ }_{34} \mathrm{CIL}, \mathrm{II}^{2} / 5,316$.

35 ABASCAL, J. M., Op. Cit. pp. 64-67 (Aelius) y 116-125 (Cornelius).

36 STYLOW, A. U., "Inscripciones latinas del Sur de la Provincia de Córdoba", Op. Cit. pp. 291-292, n. 7.

37 Estas noticias nos fueron dadas por D. Ángel Rodríguez Aguilera, director del Museo de Doña Mencía. Sus informaciones procedían de algunos de los lugareños que trabajaron en el castillo. 
muy malo. Está roto en uno de los laterales y, en general, está muy desgastado. Con dificultad se aprecian algunas de las letras de la única línea de texto que tiene.

Actualmente se conserva en el Museo histórico-arqueológico de Doña Mencía, donde la vimos y fotografiamos en 2009 (Fig. 8):

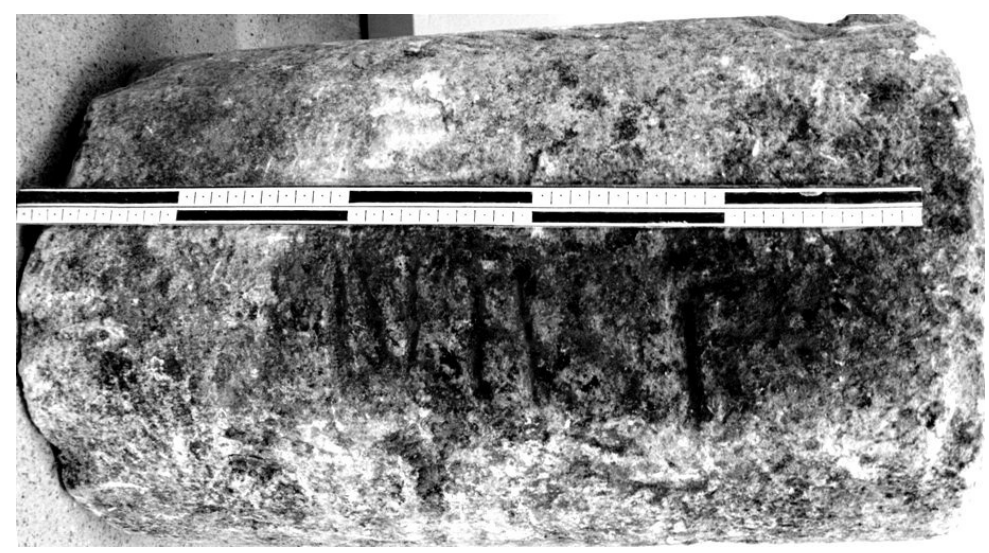

Fig. 8. Posiblemente una cupa. Museo de Doña Mencía (Fotografías de M. Pastor)

Proponemos la lectura siguiente:

BIVEL · F

- Trascripción: Bivel(i-iae)) F(ilius-a)

- Bibliografía: Inédita.

- Comentario: Las letras son librariae de trazado irregular. La incisión es poco profunda por lo que están muy desgastadas. La B prácticamente no se aprecia, pero si la I, la $\mathrm{V}$, la $\mathrm{E}$ y la $\mathrm{L}$, al igual que la $\mathrm{F}$ final; igualmente puede apreciarse una ligerísima interpuncion antes de la $\mathrm{F}$, de aquí la lectura que hemos propuesto.

En cuanto a su significado, podemos considerarlo como un monumento de carácter funerario por su forma y por el texto, a pesar de las dificultades de lectura. Es posible también que pueda tratarse de una cupa, término que se emplea para designar tumbas en forma de tonel, redondeadas o abovedadas. En este caso concreto, se ha utilizado una especie de soporte cilíndrico (fuste de columna) sin vaciado interior. Estos monumentos solían utilizarse como elementos decorativos en los espacios funerarios ${ }^{38}$. Este tipo de soportes funerarios (cupae) constituyen un

38 Sobre este tipo de monumentos, cf. ANDREU, J. (Coord.), Op. Cit. pp. $72-73$ (con bibliografía complementaria); vid. también, JULIA, D., "Les monuments funéraires en forme de demi-cylindre dans la province romaine de Tarragonaise", Mélanges Casa de Velázquez, 1, pp. 29-72; BONNEVILLE, J. N., "Les cupae de barcelona: les origenes du type monumental", Mélanges Casa Velázquez, 17, pp. 6-38; LÓPEZ, J., "Consideracions sobre les cupae i altres estructures funeráires afins", Butlleti Arqueologic, 5, pp. 21-22 y 65-103. 
tipo de monumento exento e indicativo de un enterramiento con el nombre del difunto o de un grupo familiar, como seria el caso que nos ocupa.

El nomen Bivel(ius-a) es la primera vez que se documenta en la Bética. Probablemente deriva de la raíz Bi- que se encuentra en nombres como Bibalus/Bibulus, de uso corriente en la onomástica personal romana de la Bética y de Hispania ${ }^{39}$.

Por el tipo y características de las letras podríamos fechar la inscripción y el monumento entre finales del siglo II y comienzos del III.

Finalizamos incluyendo otros documentos menos significativos, también procedentes del término de Doña Mencía y conservados igualmente en su Museo:

9. Fragmento de miliario. Al parecer se encontró en el cerro de «El Laderón». Hoy está perdido o en paradero desconocido. Lo conocemos por algunos manuscritos antiguos de los que lo copió E. Hubner ${ }^{40}$. Tan sólo conservaba las siguientes letras:

XVIII [- - ]ITIA

Por el texto, E. Saavedra y A. Fernández Guerra señalaron que podría ser de época de Trajano, concretamente del año 114, en el que el emperador desempeñó el poder de tribuno por décima octava vez (XVIII tribunitia potestate $)^{41}$, aunque no son más que conjeturas.

10. Instrumentum domesticum de época Cristiana (Fig. 9). Se trata del brazo derecho de una cruz de plata, roto por la derecha. En la parte posterior tiene inciso un pámpano con sus hojas. Apareció en el cerro de las Pozas, situado a 1 km aproximadamente al oeste de Doña Mencia, junto con monedas, fragmentos cerámicos de los siglos II al IV y un pedestal de bronce ${ }^{42} ; 0,6 / 1,5 \times(3) \times 0,1$. Las letras de 0,3 cm están grabadas entre dos líneas incisas. Actualmente se conserva en el Museo histórico-arqueológico de Doña Mencia, donde lo vimos y fotografiamos en 2009.

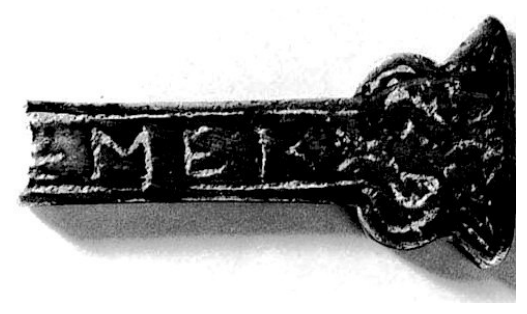

Fig. 9. Brazo de una cruz. Museo de Doña Mencía (Fotografía de M. Pastor) (Fotografías de M. Pastor)

39 ABASCAL, J. M., Op. Cit. p. 300.

40 CIL, II, 6207 (donde hace referencia a A. Fernández Guerra y a J. de Dios de la Rada y Delgado). Lo cita también SILLIERES, P., Op. Cit. pp. 144-145, n. 86 y CIL, II², p. 93 (Miliaria).

41 CIL, II², p. 93.

42 CIL, II2/5,344. 
Pueden leerse claramente las palabras:

[- - ] $] \mathrm{E} \mathrm{MEI}$

- Trascripción: [- - - miserer]e mei.

-Bibliografía: CIL, II, ${ }^{2} / 5,344$.

-Comentario: Evidentemente, se trata de una cruz cristiana, de plata, probablemente de época tardía, tal vez, del siglo VI ó VII. Recientemente se cree que es del siglo XVIII. El texto conservado formaría parte de una invocación a Dios, tal vez, Deus, o Domine miserere mei, que iría sobre tres o cuatro de los brazos de la cruz.

11. Instrumentum domesticum. Anillo de bronce (Fig. 10). Se encontró en el cerro de «El Laderón», y fue llevado al Museo Arqueológico de Madrid, donde lo vio A. Fernández Guerra, que pasó copia a E. Hübner ${ }^{43}$. Luego se perdió y está en paradero desconocido. Hübner lo describió con un dibujo y unas letras ininteligibles $^{44}$. Las letras que parecen apreciarse son las siguientes:

$\underline{\text { I Z V G T V', }}$

A O 'I’ E I V I

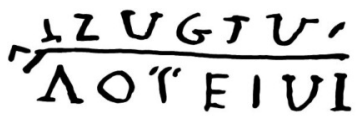

Fig. 10. Anillo de bronce. Letras de E. Hübner

$43 \mathrm{CIL}, \mathrm{II}^{2}, 4976,38$.

$44 \mathrm{CIL}, \mathrm{II}^{2} / 5,342$. 